\title{
Novel Polyimide Materials Produced by Electrospinning
}

\author{
Guangming Gong and Juntao Wu*
}

Additional information is available at the end of the chapter

http://dx.doi.org/10.5772/53459

\section{Introduction}

\subsection{Electrospinning}

Due to one-dimentional nanostructures' unique properties and their intriguing applications, there has being a great demanding on the technologies which are able to produce such structures. Among the applicable strategies, electrospinning seems to be the simplest approach to generate continues nanofibers with ultrathin and uniform diameters ( $\mathrm{Li} \& \mathrm{Xia}, 2004$ ). Electrospinning is a spin technology which involves the use of a high voltage, usually directcurrent, to trigger the formation of a liquid jet (Li \& Xia, 2004; Greiner \& Wendorff, 2007).

\subsubsection{The Mechanism of Electrospinning}

Briefly, the mechanism of electrospinning is often to be explained as the collaborative effects of electrostatic repulsion by the accumulated charges on the surface of polymer solution and the Coulombic force exerted by the external electric field ( $\mathrm{Li} \&$ Xia, 2004; Greiner \& Wendorff, 2007). A illustrative figure may help the understanding of it. As can be seen in Figure 1, polymer solutions are added in a syringe with a metal spinneret connected on its tip. Driven by a syringe pump or gravity, the solution starts to flow out. When a high voltage (usually in the range from 1 to $30 \mathrm{kv}$ ), the pendent drop of the solution will be highly electrified. During this process, the liquid drop will be shaped into a cone-like object, as known as "Taylor Cone". And the charges on the surface will repulse each other, overcome the surface tension of the solution until reaching a certain threshold. Then an ejection of solution is formed. Driven by the mighty electric field, the liquid jet undergoes a mighty stretching force, elongating the jet, thinning the diameter to a certain range. While during this period, the solvent evaporates intensively. Thus, the electrospun polymer fibers are formed. And a typical SEM photo of the electrospun fiber is illustrated in the insert of 
Figure 1. Electrospinning is able to produce fibers whose diameters ranged from tens of nanos to a few microns. And a great amount of polymers are reported to be successfully spun into fibers via this technology. Based on the experimental data and electrohydrodynamic theories, several groups have built mathematical model to describe behaviors of electrospinning. Further information is not explained here, if interested, one can refer to these literatures: (Reneker, Yarin \& Fong, et al., 2000; Yarin, Koombhongse \& Reneker, 2001; Hohman, Shin \& Rutledge, et al., 2001, etc.)

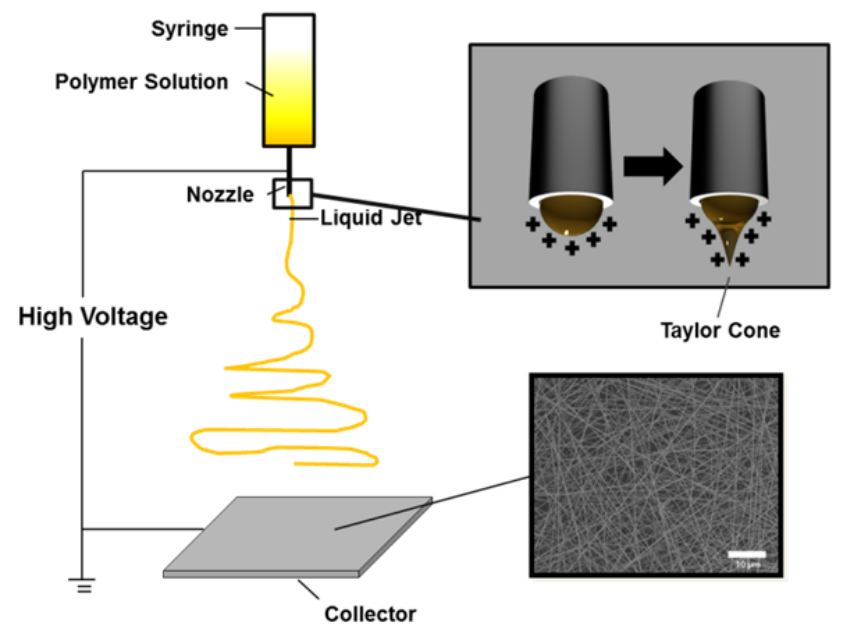

Figure 1. An illustration of a typical electrospinning set and the formation of the liquid jet (in enlargement). The inserted is an SEM picture of nowoven PI mat prepared in our laboratory.

\subsubsection{A Brief History of Electrospinning}

The first patent that describe the operation of electrospinning appeared in 1934 (Formalas, 1934), when Formalas disclosed an apparatus for producing polymer filaments by taking the advantage of electrostatic repulsions between surface charges. It has been almost 80 years since the patent but electrospinning existed only as a theory in people's mind until 1990s. (Li \& Xia, 2004) During that period, there were only few reports on ES. Its value was not fully attended so that no further development was made. But in the beginning of 1990s, by the efforts of several research groups, especially the ones leaded by Prof. D. H. Reneker and Prof. G.C. Rutledge, people demonstrated that ES was able to produce a great amount of polymer fibers. A large campaign of polymer ES researches was waged. (Wikipedia, Online) The timely demonstrations popularized the term "electrospining" in literatures that we see today.

\subsubsection{Development and Applications}

The diameters of polymer fibers produced by electrospinning were able to reach an incredible small range. Compared to traditional polymer fibers, ES fibers have greater 
specific areas and aspect ratio (Li \& Xia, 2004; Greiner \& Wendorff, 2007). Electrospinning is a method to produce not only pure fiber structures, but also many other morphologies. By tuning electrospinning parameters (e.g. electric field strength, viscosity of spinning solution, solvents, etc.) and/or choosing different ES methods, controllable morphologies of ES products can be achieved. Owing to the world wide collaborative efforts, so far, people are able to electrospin pearl-necklace-like beaded fiber structures (Greiner \& Wendorff, 2007), highly porous fiber structures (Bognitzki et al., 2001), grafted fiber structure (Hou H, Reneker D H, 2004; Chang Z, 2011), hollow interior micro tubes (Li, Wang \& Xia, 2004; Zhao, Cao \& Jiang, 2007), wire-in-tube structures (Greiner \& Wendorff, 2007), What's more, twisted fiber bundles, golfball-like micro particles and multi-chambered hollow spheres (Chen et al., 2008) are also able to be generated via this technique. These fibrous structures and particles obtained by electrospinning have some unmatchable properties, like the

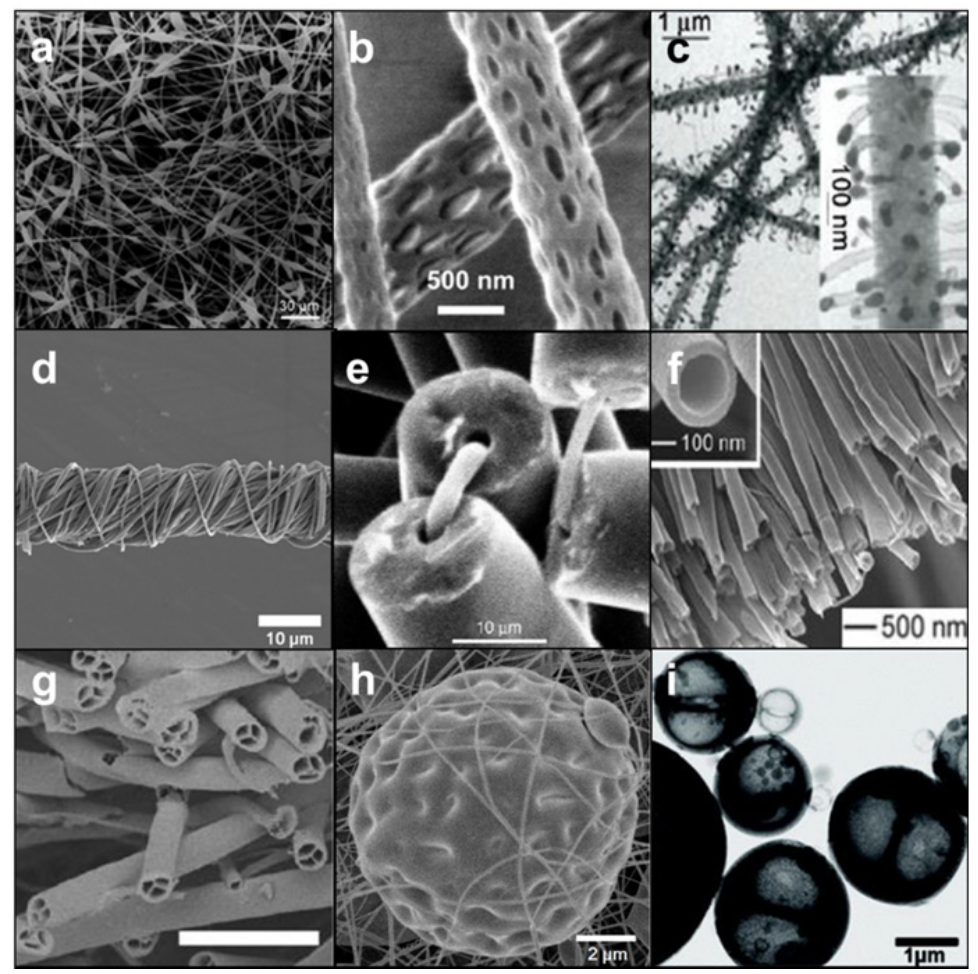

Figure 2. Morphologies of electrospun products: a) pear-necklace like fibers. Copyright $(2007$ WILEY$\mathrm{VCH}$, reused with permission; b) highly porous fibers. Copyright ( 2001 WILEY-VCH, reused with permission; c) grafted fibers. Copyright (C) 2004 WILEY-VCH, reused with permission; d) a bundle of electrospun PI micro rope (Gong \& Wu, unpublished work); e) wire-in-tube fibers. Copyright $(2007$ WILEY-VCH, reused with permission; f); aligned micro tubes. Copyright $@ 2$ 2004, American Chemical Society, reused with permission; g) multi-channel tubes. Copyright $@$ 2007, American Chemical Society, reused with permission; $\mathrm{h}$ ) golfball-like spheres (Gong \& Wu, unpublished work); i)multi-chamber spheres. Copyright $\odot$ 2008, American Chemical Society, reused with permission 
extremely long length, high surface area, complex pore structure, alignment on the molecule level, etc. Such properties enable the electrospun materials possessing the potential values in applications and research fields, e.g. templates, filter and textile, catalysis and enzyme carriers, nanofiber reinforcement, medical applications, and surfaces with special wettability, etc. Due to the space constraints, detailed instances are not discussed here. The further introductions and examples, if one is interested, can be reffered to these literatures: (Li \& Xia, 2004; Greiner \& Wendorff, 2007; Lu, Wang \& Wei, 2009)

\subsection{Polyimide (Ding, 2011)}

Polyimide (PI) is a polymeric material which contains imide rings in their molecule backbones. PI is mainly obtained through a two-step method: fragrant diamine and dianhydride undergo a condensational polymerization and then a thermal/chemical imidization, to afford PI, illustrated in Figure 3. The representative PI is Kapton ${ }^{\circledR}$, developed and commercialized by Dupont ${ }^{\mathrm{TM}}$ in 1960 and 1965, respectively. This golden film presents good mechanical properties and high thermal stability. After 50 years, Kapton ${ }^{\circledR}$ is still a leading material in thermal tolerable materials. After Kapton ${ }^{\circledR \prime} \mathrm{s}$ success, a series of fragrant polyimides were developed and accepted by the market. PI's wide popularity is due to its comprehensive performances: wide applicable temperature range, good mechanical, electrical properties and bio-compatability. Besides, PI's broad molecular designing window also guarantees its rapid development: by modifying molecular structures of diamine and dianhydride, different kinds of soluble and thermal shapeable PI were prepared. After a long term of research and developing state, PI is now extensively employed in the aerospace and aviation industries and in the microelectronic and electric fields as advanced packaging and insulating materials. With the rapid development of these advanced industry, novel PI materials with high performances and new functions are usually required.

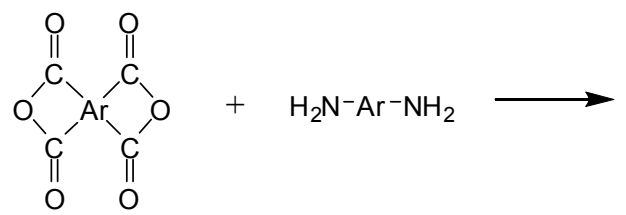<smiles></smiles>
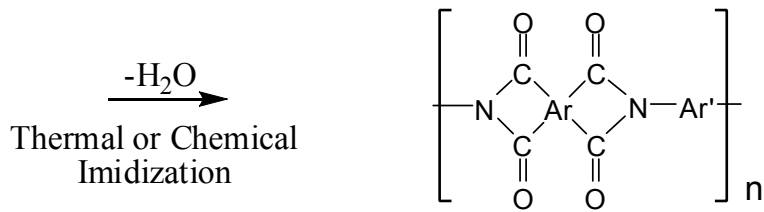

Figure 3. A two-step PI synthetic route 


\subsection{Electrospun polyimide materials}

In 1996, Reneker referred that PI nano fibers are obtainable via electrospinning (Reneker \& Chun, 1996). In 2003, Nah et al. described in details the method to produce PI nano fibers (Nah, Han \& Lee, 2003), declared the successful preparation of ultra-thin PI fibers. The morphology of the PI fibers is shown in Figure 4. The successful preparation was a milestone that it was when a polymer with comprehensive high performances encountered a versatile and effective method for fiber producing. Their combination afforded a series of results with both scientific amd industrial values. Herein, this chapter summarized and categorized the works of electrospun PI materials since 2003. In the meantime, some outlooks of this research field were also given.
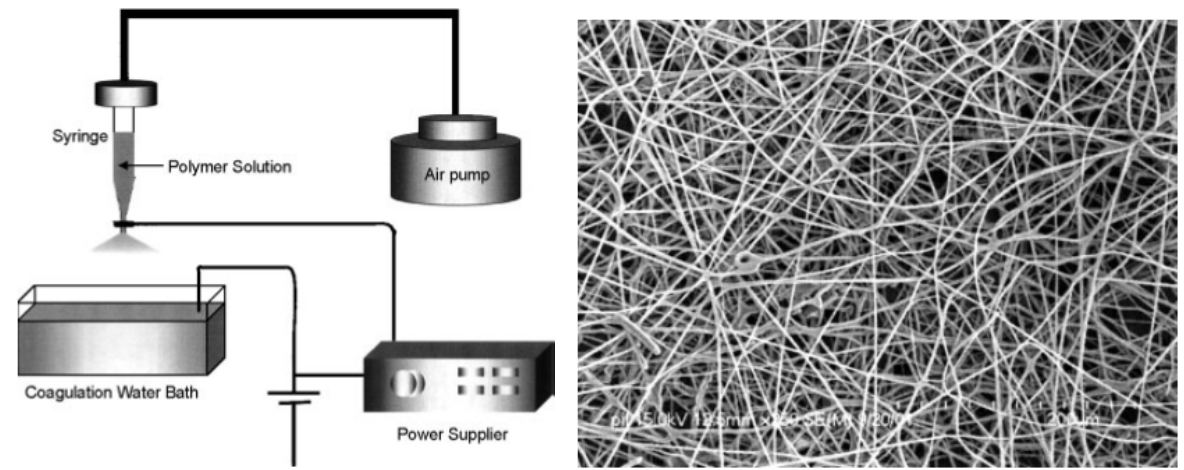

Figure 4. Nah et al. obtained PI fibers by electrospinning for the first time, left: the electrospinning set; right: SEM of PI mat. Copyright (C) 2003 WILEY-VCH, reused with permission

\section{Carbon fiber precursors: the preparations of electrospun nano PI fibers}

Carbon fibers produced from traditional fibers possess high tensile strength and modulus of elasticity. People wish to be able to produce thinner carbon fibers from electrospun polymers after the electrospinning technique was born. Due to the ultra-thin diameter of the electrospun fibers, compared to the ordinary art of carbon fibers, the ultra-thin carbon fibers produced from electrospun fibers possess much higher specific area. Such property is in favor of super capacitors or the carrier of the catalyst (Li \& Xia, 2004; Dong, 2009). The researchers also found that, interestingly, carbon fibers produced from electrospun PI fibers possess higher conductivity than some ordinary materials using the same method. As a result, ressearchers attempted to get carbonized products with higher performances from the electrospun PI fibers. Some have achieved initial results and they are introduced as follow.

In 2003, Yang et al. first reported the method of carbon fiber preparation by carbonizing the electrospun PI fibers (Yang et al, 2003). They discussed the parameters for the PI electrospinning, pointing out the proper concentrations and viscosity of the solutions, as well as the voltage and electric field. Their job provided precious experience for the followup works. In their work, 4,4'-oxydianiline (ODA) and pyromellitic dianhydride (PMDA) 
underwent a condensational polymerization to form polyamic acid (PAA), the precursor of PI. Certain amount of PAA with certain concentration ( by weight) was added into the syringe to be electrospun into fiber samples with diameters ranged from 2 to $3 \mu \mathrm{m}$. After thermal imidization, the diameters shrank to $1 \sim 2 \mu \mathrm{m}$. After the final carbonizing, carbon fibers were obtained. Yang et al. discovered that the conductivity of the samples increased as the carbonizing temperature rose. The conductivity reached $2.5 \mathrm{~S} / \mathrm{cm}$ after carbonized under $1000^{\circ} \mathrm{C}$, significantly higher than the one of which produced from PAN fibers $(1.96 \mathrm{~S} / \mathrm{cm})$ treated under the same conditions and procedures. An SEM photo of the carbonized mat is shown in Figure 5.

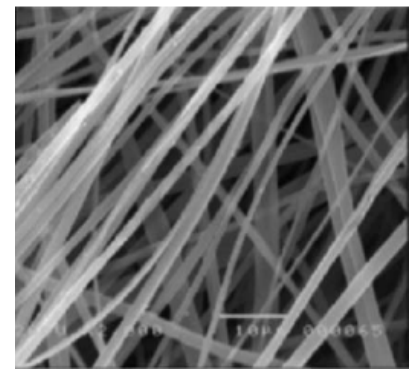

Figure 5. SEM of PI nonwoven mat after carbonization. Copyright (C) 2003 Elsevier, reused with permission

In 2004, Kim et al. reported the preparations of carbon fibrous electrode with high performance via electrospinning of PI (Kim et al, 2004). The nonwoven mat they prepared was made into electrode after carbonizing at $2200^{\circ} \mathrm{C}$, the conductivity was as high as $5.26 \mathrm{~S} / \mathrm{cm}$, detected by the four-probe method. The specific capacity could reach $175 \mathrm{~F} / \mathrm{g}$ at most. Cyclic Voltammetry and Alternating Current Impedance Spectra both demonstrated its excellent performance. Due to the super thin diameters of each single fiber, a huge amount of micro and nano gaps were introduced into the body of the electrode. As a result, the specific area of the electrode is incredibly high, $1453 \mathrm{M}^{2} / \mathrm{g}$ (detected by BET test), which is unmatched by ordinary electrode. The high porosity, specific area and conductivity of the ultra-thin carbon fiber produced from electrospun PI, brought about high specific capacity and high reaction reversibility to such kind of electrodes, enabling its popularity in the preparations of high performance electrodes, super capacitor and energy storing.

Chung et al. reported a new way to produce carbon fibers in 2005 (Chung et al, 2005). The soluble PI, Matrimid ${ }^{\circledR}$ 5218, was directly electrospun into nonwoven mat and then carbonized. In the electrospinning process, the authors added a certain amount of $\left[\mathrm{CH}_{3} \mathrm{COCH}=\mathrm{C}\left(\mathrm{O}^{-}\right) \mathrm{CH}_{3}\right]_{3} \mathrm{Fe}$ as additives. In the following characterizations, XRD, Raman Spectra, SEM, TGA all demonstrated that $\left[\mathrm{CH}_{3} \mathrm{COCH}=\mathrm{C}\left(\mathrm{O}^{-}\right) \mathrm{CH}_{3}\right]_{3} \mathrm{Fe}$ might promote the carbon yield, enlarge the crystal dimension, increase the thermal stability at the same time.

In 2007, Xuyen et al. for the first time discussed how the electrospinning parameters would affect the PI fibers quantitatively (Xuyen et al., 2007), part of the data is illustrated in Figure 6. Further they discussed the relationship between the diameters of PI fibers and the 

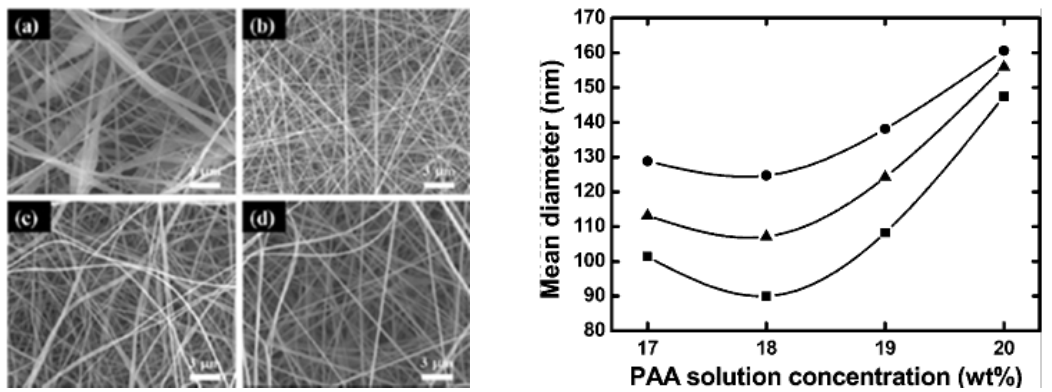

Figure 6. The study of parameters on the PAA electrospun diameters. Left: fiber morphology affected by the amount of TEA while electrospinning, a) $0 \mathrm{wt} \%$, b) $1 \mathrm{wt} \%$, c) $3 \mathrm{wt} \%$, d) $5 \mathrm{wt} \%$; right: fiber diameters affected by concentrations of PAA solution. Copyright (c) 2007, American Chemical Society, reused with permission

conductivity of their carbonized products. The parameters they referred included the amount of catalyst (triethyl aminde, TEA), the electric field and concentrations of the solution (wt $\%$ ). This research revealed that: in some range, fiber diameter decreased when the amount of TEA increased; the fiber diameter increased while the concentration increased; the critical electric field was quadratic to the molar mass. According to the authors' explanations, firstly, TEA promoted the polymerization of ODA and PMDA, leading to a high viscosity during the synthesis, which stabilized the Taylor Cone, inhibited the variation of the fiber diameter during electrospinning; Secondly, at the same feeding rate, while the concentration increased, the amount of PAA increased in the electrospun sample, leading to the expansion of the fiber diameters; At last, due to the quadratic relationship (Jones \&Richards, 1999) between surface tension of the PAA solution and the molecule weight, while the critical electric field was directly linked to the surface tension (Taylor, 1969), as a result, the critical electric field and molecule weight could be described as follows:

$$
E_{\mathrm{c}}^{2}=563 \bullet\left[1-\left(\frac{57178}{M_{n}}\right)^{1.8}\right]
$$

where $E_{c}$ is the critical electric field and $M_{n}$ is the number-average molar mass. Experimental data fitted the equation well. During the research, by controlling the diameter of the fibers and the pressure during the carbonization, Xuyen et al. discovered that the conductivity increased when the diameter decreased and the pressure increased. The maximum value they obtained was as high as $16 \mathrm{~S} / \mathrm{cm}$, and the fiber diameter was only $80 \mathrm{~nm}$. Compared to the formal reported ones (Yang et al, 2003; Kim et al, 2004), the fiber diameter was sharply reduced while the conductivity was greatly enhanced, demonstrating the great advantages of electrospinning in carbon fiber preparations.

\section{PI nanocomposites prepared via electrospinning}

During the beginning of electrospinning process, the solution jet bears huge shear force and stretching force, so that the fiber diameter reaches the nano range. Then three factors will 
limit the agglomerations of the nano particles in electrospun nanocomposites. The three factors are: the constrain of the fiber diameter, mighty electric force and the surface tension of the polymer solution as well. In addition, during the end of the process, due to the fast evaporation of the solvents, the polymer fiber will be solidified rapidly as if the liquid was "frozen". Once "frozen", the reagglomerations of the nano particles will be greatly prohibited. These factors enable electrospinning a versatile and effective method for the dispersion of nano particles in the polymer body (Behler et al., 2009). There have been a great amount of reports on this technique about the dispersions of functional nano particles into polymer fibers, involving the functions of catalyst, thermal conductivity, light adsorption and bio characteristics, etc. Detailed examples are not discussed here.

What's more, PI is a intrinsically tough resin with comprehensive high performance as we introduced above. By electrospinning, we may be able to disperse a variety of functional particles into its body in order to prepare PI materials with high performance and certain functionalities. Such idea is worth of great scientific and industrial values because it might realize the functionalization of PI and in the meantime, expand PI's applications. Several research groups are carrying out such works and a few results are mentioned as follow.

Zhang et al. reported the successful preparation of nano Ag particles/PI fiber composite in 2007 (Zhang et al., 2007). Due to the excellent optical, electrical, catalytic and anti-microorganism properties, nano Ag particles are widely applied in composite preparations. The $\mathrm{Ag} / \mathrm{PI}$ composite that Zhang et al. prepared are potentially applicable in optics and catalysts.
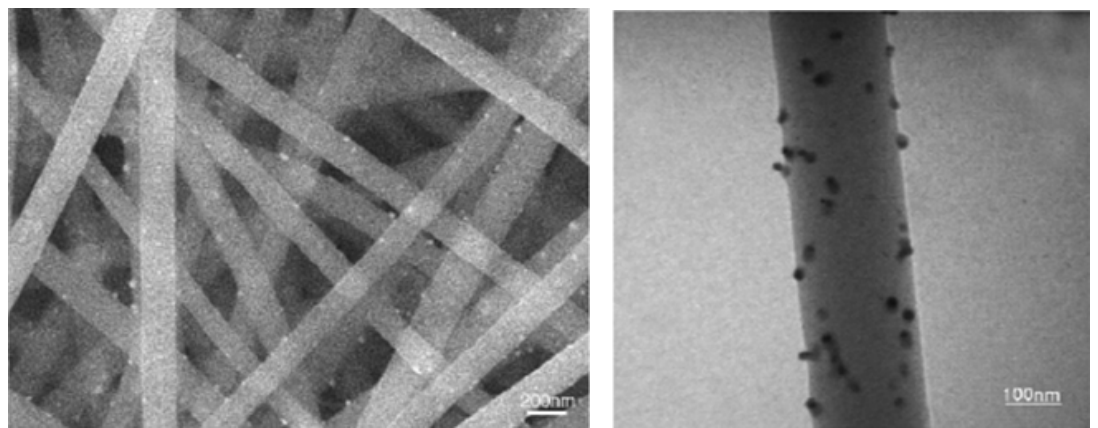

Figure 7. SEM (left) and TEM (right) of nano Ag/PI fiber composites. Copyright $\odot 2007$ Elsevier, reused with permission

In 2009, Chen et al. reported the successful preparation of multi-wall carbon nanotubes (MWCNTs)/PI composite via electrospinning (Chen et al., 2009). The aim of the research was to add MWCNTs into PI fiber systems to enhance the mechanial properties of the composite. The key problem is to disperse the MWCNTs homogeneously. However, MWCNTs are not compatable with the PAA solution. To increase the compatibility of the nano filament with the polymer, MWCNTs were treated by high concentrations of nitrate, in order to introduce oxygen-contained groups onto their surfaces. Then after in-situ polymerization, the MWCNTs/PAA was then electrospun into nano fibers. During the ES process, a special set was used to introduce alignment into the fiber belt. After the final thermal imidization, the 
mechanical properties of the aligned fibrous MWCNTs/PI composite were examined. Compared to the neat PI fiber belt, the mechanical performances of the composite were obviously more advantageous: the yield strength, tensile strength and tensile modulus were 200.9 $\mathrm{MPa}, 239.7 \mathrm{MPa}$, 2.56 GPa, respectively. And the elongations at break could reach as high as $90.5 \%$. At the same time, the thermal stability of the composite was greatly enhanced. The TEM demonstrated the well dispersion of the filament in PI matrix and the filament were well aligned along the fiber direction. The alignment of these filament might contributed to the shearing and stretching force of the mighty electric field.
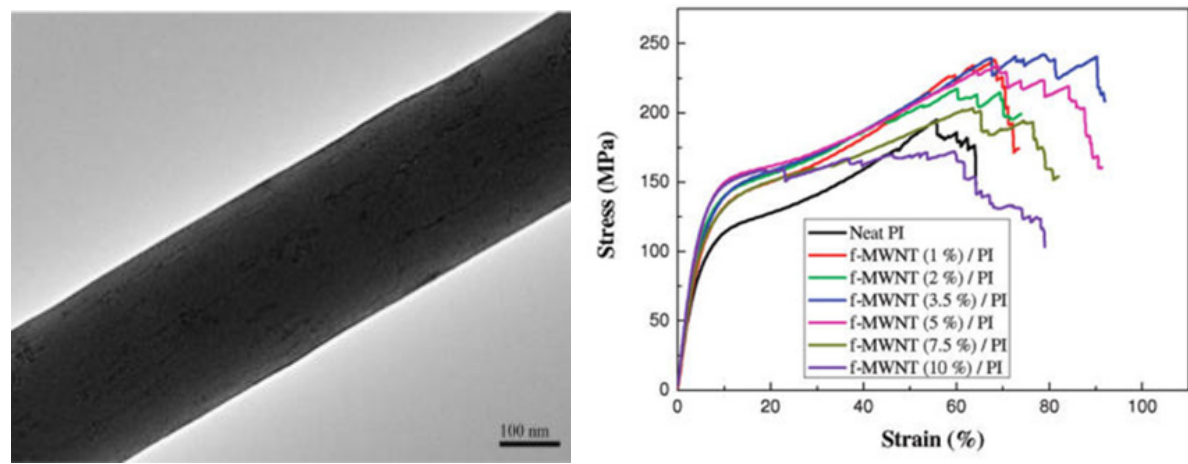

Figure 8. TEM of nano-carbon tubes/PI fiber composites (left) and its stress-strain curve (right). Copyright (C) 2007, American Chemical Society, reused with permission
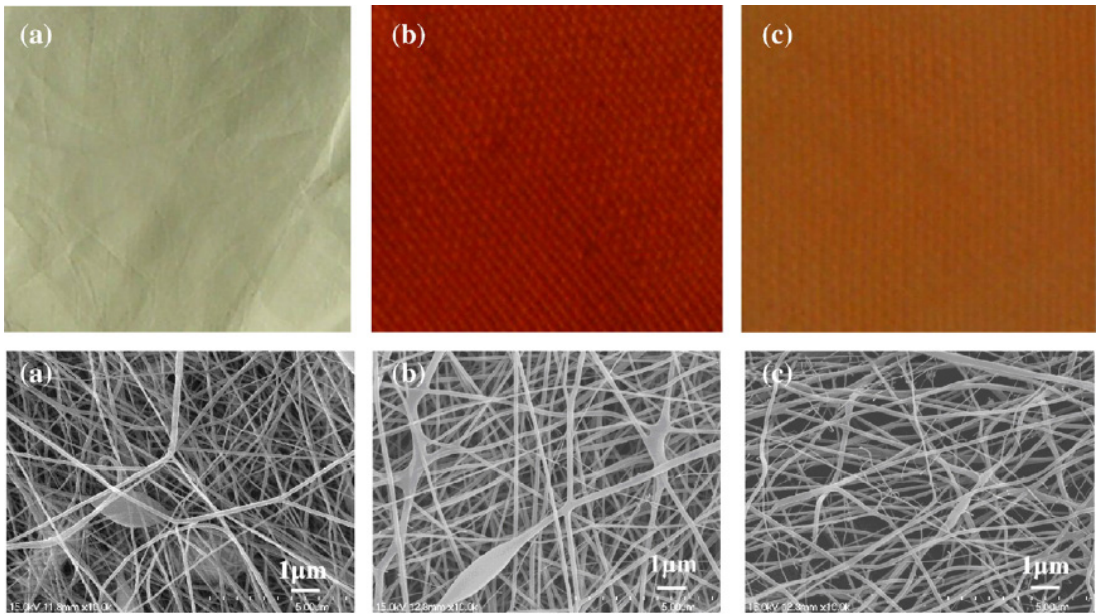

Figure 9. Images of PI-(hemicyanine dye) hybrid. The upper row are digital pictures of a): Pure PAA nonwoven mat, b):PAA-(hemicyanine dye) hybrid nonwoven mat, c): the hybrid mat after imidization; the lower row are the corresponding SEM images. Copyright @ 2009 Elsevier, reused with permission 
In 2010, the methodology of preparations of PI composite with magnetic effect was reported by Zhu et al (Zhu et al., 2010). In their work, Fe-FeO nano particles with core-shell structures were doped into soluble PI, Matrimid $5218^{\circledR}$. The inorganic particles were well compatible with the PI matrix without further treatment. The research found out that the ES has no major negative effects on the saturation magnetization of the particles, while after the ES process, the coercivity was increased from 62.3 Oe (neat particles) to 188.2 Oe (composite), which meant the composite's anti-magnetic ability was improved. Zhu et al. pointed out that such improvement was triggered by the uniform dispersion of the inorganic particles which would lead to the weakening of the dipolar interactions between each and every one.

Moreover, there were other reports on the electrospun PI composite: Qin et al. reported the fluorescent performance of electrospun PI web mixed with hemicyanine dye (Figure 9), pointed out that the nano fiber structure were helpful to the fluorescence (Qin et al., 2009); Cheng et al. reported the preparation of nonwoven PI/silica hybrid fabrics by combining electrospinning and controlled in situ sol-gel techniques, their product had better mechanical and thermal properties than ordinary PI electrospun fabrics (Cheng et al., 2009).

\section{Novel light and tough electrospun PI fabrics}

Due to PI's excellent mechanical properties, it is widely applied in military industry, engineering, aeronautices and astronautics. The most intriguing point is that electrospinning is able to improve PI's mechanical properties. The molecule chains will be greatly constrained within the nanofibers. As a result, the crystallization area is increased, leading to the enhancement of the nanofiber's mechanical property. Now that nano PI fibers are achievable via electrospinning and such fibrous PI materials with lighter weight and excellent performances are intriguing, we consider this research of great importance and several scientific outcome are shown below.

In 2006, Huang et al. prepared PI nonwoven mat with considerable mechanical properties (Huang et al., 2006). 3,3',4,4'- biphenyl dianhydride (BPDA) and P-phenylenediamine (PDA) were polymerized into PAA with high molar mass. After ES and imidization, the mat's tensile strength and Young's modulus were detected as $210 \mathrm{MPa}$ and $2.5 \mathrm{GPa}$. Compared to the formerly reported mats (Cheng et al., 2009; Kim et al., 2004; Yang et al., 2003), this product exhibited higher mechanical performances. In this work, they mentioned the high molar mass guaranteed the high performance. In the same year, they reported a new way (Huang et al., 2006) to fabricate PI mat with higher mechanical properties. In that work, a rotating wheel with a $8 \mathrm{~mm}$ wide edge was utilized to collect the electrospun PI fibers. The fibers in the as-prepared PI fiber belt were highly aligned and along the align direction, the tensile strength and Young's modulus reached as high as $664 \mathrm{MPa}$ and $15.3 \mathrm{GPa}$, respectively.

In 2008, for the first time, the mechanical property of a single BPDA-PDA nano fiber was reported (Chen et al., 2008). The authors used a square frame to collect a few strands of PI fibers by waving across through the space between the spineret and collector rapidly several times. Then special care were taken to mount one single fiber into the micro tensile testing 
machine. In the described details, the mechanical properties of both PAA and PI single fibers with diameters aroung 300nm were characterized: the data of PAA fiber was $766 \pm 41 \mathrm{MPa}$ in tensile strength, $13 \pm 0.4 \mathrm{GPa}$ in tensile modulus, $\sim 43 \%$ in elongation at break; the data for the PI fiber was $1.7 \pm 0.12 \mathrm{GPa}$ in tensile strength, $76 \pm 12 \mathrm{GPa}$ in tensile modulus, $\sim 3 \%$ in elongation at break. According to the authors, the excellent mechanical performance stemmed from the high alignment of the macromolecules in the fiber structure, which was confirmed by Wide-angle XRD.

In this series of works, the PI matrix was synthesized from BPDA and PDA. In such kind of polymer chains, there are few flexible groups, which will make the material hard and brittle. This explains why those PI fiber belt possess low elongation at break and unsatisfactory toughness and flexibility. To improve the toughness and flexibility of the electrospun PI materials, in 2008, Chen et al. added 4,4'-oxydianiline (ODA) into the former system (BPDA+PDA), to form block copolymers (Chen et al., 2008). Similar to the methods that have been discussed before (Huang et al., 2006), the copolymers were also electrospun into aligned fiber belt but with ameliorated elongation properties. Due to the introduction of flexible groups, the elongation at break of the copolymer could reach $\sim 20 \%$, and the tensile strength and modulus were $1103 \pm 61 \mathrm{MPa}, 6.2 \pm 0.7 \mathrm{GPa}$. Such a PI belt with $7.5 \mathrm{~mm}$ wide and $1.5 \mu \mathrm{m}$ thick could bear a $10 \mathrm{~kg}$ load, illustrated in Figure 10.

In 2009, they reported a PI fibrous material with even higher stretching ability (Cheng et al., 2010): the elongation at break could reach as high as $200 \%$. This PI was synthesized from BPDA and 2-Bis[4-(4-Aminophenoxy)Phenyl] Hexafluoropropane (6FBAPP). 6FBAPP contains more flexible "-O-“ groups, so such PI materials are more pliable but not strong enough. The BPDA-6FBAPP exhibited 308 $\pm 14 \mathrm{MPa}$ in tensile strength and $2.08 \pm 0.25 \mathrm{GPa}$ in tensile modulus. From the results of the series of researches, in summary, the molecule

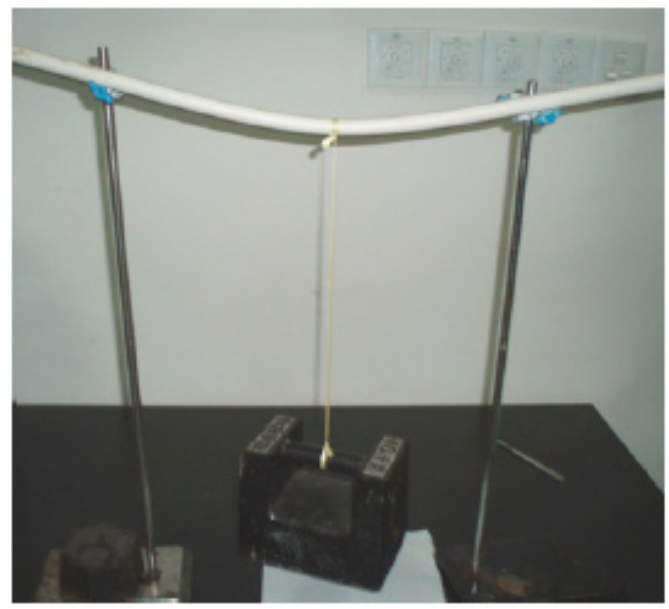

Figure 10. A photograph of the electrospun copolyimide nanofiber belt hanging a weight of $10 \mathrm{~kg}$; the belt has $7.5 \mathrm{~mm}$ width and $12 \mu \mathrm{m}$ thickness. Copyright $\odot 2008$ IOP Publishing Ltd, reused with permission 
structures greatly affect the macroscopic property of electrospun PI materials. The molecule structures are summerized in Table 1. In addition, the molar mass and the aligment of fibers are also vital keys to such kind of material.

Dianhydride

Table 1. A list of molecule structure of aromatic anhydrides and amines referred in Section 4

The PI fabrics discussed above were only aligned in one certain direction, in other words, the mechanical property could only be presented anisotropically. So far, constrained by the development of patterning technology of electrospinning, there have been no reports on multi-directional aligned PI fabrics with isotropic high mechanical performances. However, in 2008, Carnell et al. reported a novel way to fabricate perpendicularly aligned PI fabrics (Carnell et al., 2008). They applied a needle-like auxillary electrode opposite to the spinneret, and used a metal roller to collect the fibers. The set for electrospinning the aligned fibers is shown in Figure 11. In their work, when negative high voltage was applied on the auxiliary electrode, because of the guide and confinement of the concentrated electric field lines, the instability of the spin jet was greatly suppressed, leading to fine alignment in one certain direction. What's more, by re-place the collector, the aligned fibers can be vertically

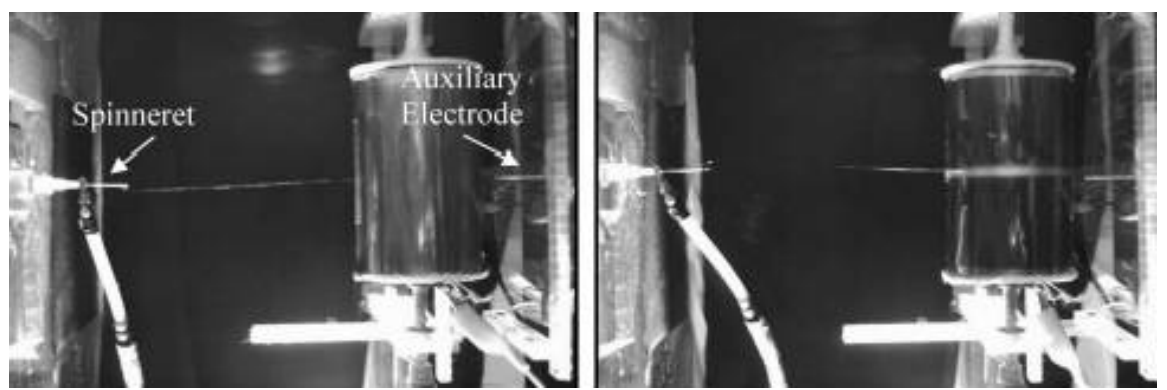

Figure 11. The set for electrospinning the aligned fibers. Copyright ( 2008, American Chemical Society, reused with permission 
staggered (shown in Figure 12), to form fabrics with double-directional alignment. Such technique could improve the continuity and yield of the aligned fibers, and especially it expanded the dimensions for the alignment of electrospun nano fibers.

In 2010, Lee et al. reported a method to optimize the fiber morphology and mechanical property (Lee et al., 2010). During the ES process of co-Poly-(amide)-imide (PAI), the fibers were soaked in glycerine. And during the imidization, the fibers were stretched. After these procedures, the PAI fibers presented smoother sectional shapes, better alignment and as a result, as we have discussed above, the mechanical property should be improved but not discussed in that article. In fact, the stretching imidization is a critical step for enhancing the mechanical performance of PI material. So the stretching imidization is also expected to be one effective way to improve the characteristics of the electrospun PI fibers.
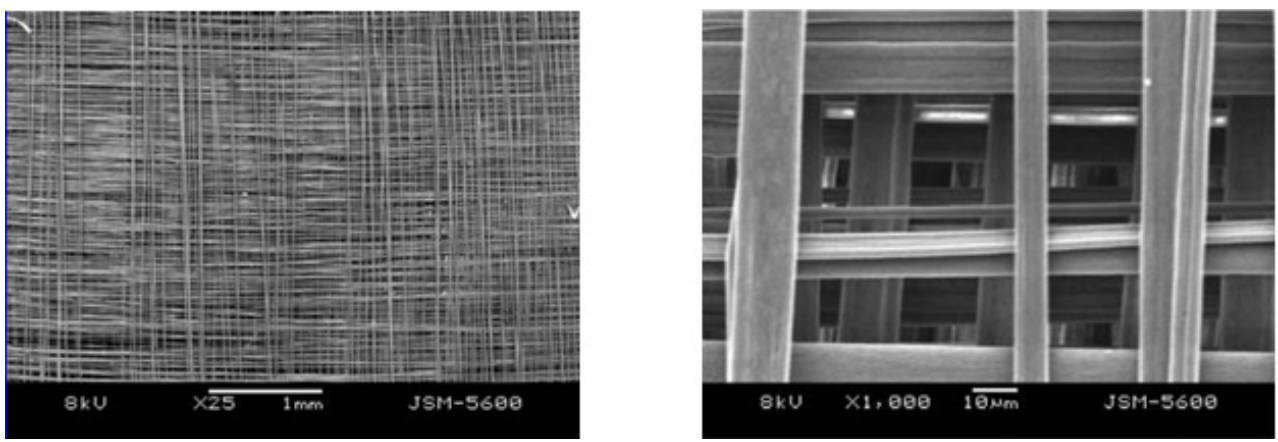

Figure 12. Horizontally and vertically aligned PI nonwoven mat prepared through the way that reported by Carnell et al. Copyright ( () 2008, American Chemical Society, reused with permission
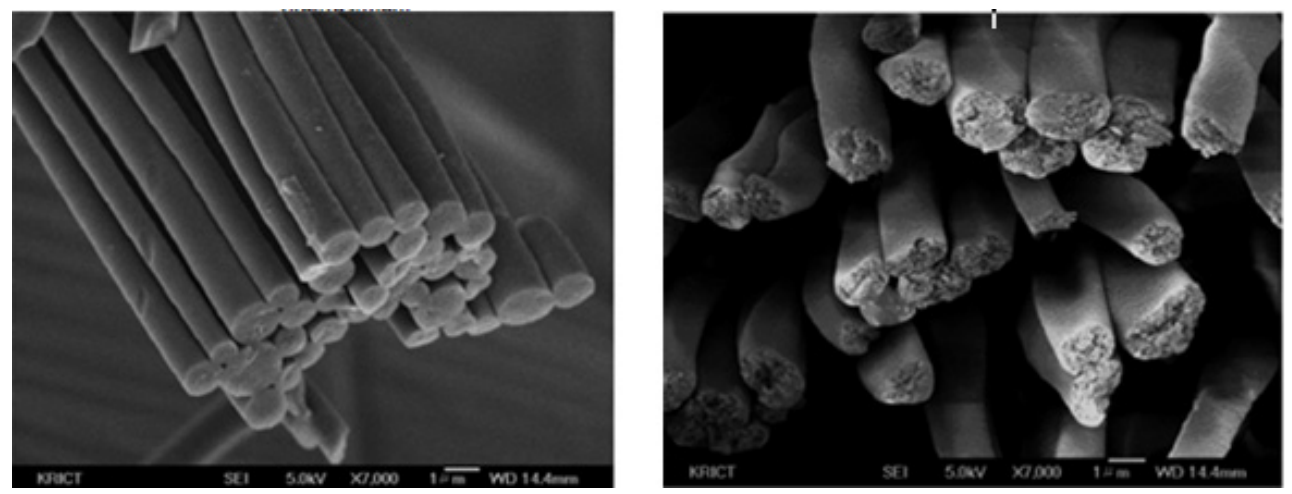

Figure 13. SEM photos of the cross-sectional surfaces of continuous copolymer nanofiber bundles: imidized under tension after glycerol pre-treatment. Copyright (C) 2010 WILEY-VCH, reused with permission 


\section{Others}

In recent years, reports on other functional electrospun PI fibers thrived. Herein we have chosen some typical works to discuss. In 2007, Liu et al. reported a new way to produce PI material with ultralow dielectric constant $(k)$ via electrospinning (Liu et al., 2007). The traditional $k$ of a piece of PI film, e.g. Kapton ${ }^{\circledR}$, is about 3.5. However, the electropsun PI mat's $k$ could reach as low as 1.5 according to Liu et al. The theory could be explained as this: during the process of ES, due to the nano size of the fiber diameters, a great amount of mesoscopic gaps would be introduced into the body of the PI mat. As the fiber diameter decreased and/or the aspect ratio increased, the amount of gaps increased, making the inner space of the mat more and more similar to pure air, leading to the decrease of $k$ of the PI mat.

In 2008, Lv et al. reported that they used two kinds of diamines: diaminotetraphenylporphyrin and ODA, copolymerized with PMDA to form porphyrin modified PAA (Lv et al., 2008). Then it was electrospun and imidized into fluorescent nonwoven mat. The characterizations, including UV-vis spectra, fluorescence spectra, ${ }^{1} \mathrm{H}$ NMR spectra, TGA, demonstrated that the concentration of porphyrin, the nanostructures were the main factors which would influence the quantum yield and the fluorescent property. The higher the concentration is and the thinner the fiber diameters are, the higher the quantum yield is and the stronger the fluorescence is. In addition, the existence of heavy metal ions, e.g. $\mathrm{Hg}^{2+}$, will lead to fluorescence quenching which can be detected by naked eye. So that this PI mat can be used as the sensor of heavy metal ions.

In 2009, Chang et al. utilized the technique of "click chemistry" to successfully link alkynylterminated polymethyl methacrylate (PMMA) onto the surfaces of the electrospun PI nanofibers (Chang et al., 2009). The route was illustrated in Figure 14. The extremely high specific areas of the nanofibers were the key to the success and Chang's work was the first attempt to graft other polymers onto the PI surface, provided a novel idea to the surface modification of PI.

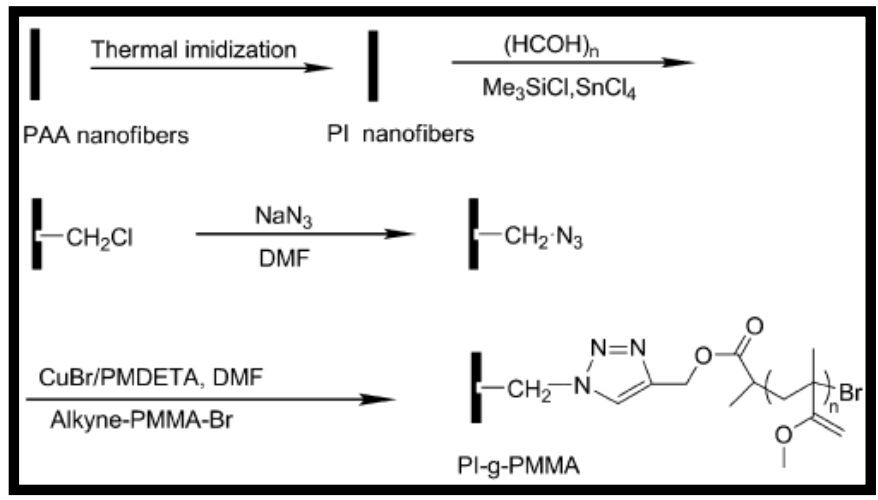

Figure 14. Synthetic route for the "Click Chemistry" for preparing the grafted PI fibers. Copyright $\odot$ 2009, American Chemical Society, reused with permission 


\section{Conclusion}

In the past few years, researchers pave their way through the field of the preparations of electrospun PI materials. The combination of the facility and effectiveness of electrospinning and the high performance of PI give birth to a series of novel PI materials with hierarchical constructions and multi-functionalization. In the coming researches of PI materials, the electrospinning way is worthy of noting and will attract more and more research interest. And we hope that novel PI materials produced by electrospinning can be used in the field of civil engineering, electrics and aero\&astronautics in the near future.

\section{Author details}

Guangming Gong and Juntao $\mathrm{Wu}^{*}$

Key Laboratory of Bio-Inspired Smart Interfacial Science and Technology of Ministry of

Education,School of Chemistry and Environment, Beihang University, Beijing 100191, PR China

\section{Acknowledgement}

This work is financially supported by the National Natural Science Foundation of China (No. 51003004), the National Research Fund for Fundamental Key Projects (No. 2010CB934700, 2012CB933200), the Scientific Research Foundation for the Returned Overseas Chinese Scholars, State Education Ministry and the Fundamental Research Funds for the Central Universities.

\section{References}

Behler, K.; Stravato, A.; Mochalin, V.; Korneva, G.; Yushin, G. \& Gogotsi, Y. (2009). Nanodiamond-Polymer Composite Fibers and Coatings. ACS Nano, Vol. 3 No. 2, (Feb., 2009), pp. 363-369, ISSN: 1936-0851

Bognitzki M, Czado W, Frese T, Schaper A, Hellwig M, Steinhart M, Greiner A, and Wendorff J H, (2001). Nanostructured Fibers via Electrospinning, Adv. Mater., Vol. 13, No. 1, (Jan, 2001), pp. 70-72, ISSN 1521-4095

Carnell, L. S.; Siochi, E. J.; Holloway, N. M.; Stephens, R. M.; Rhim, C.; Niklason, L. E. \& Clark, R. L. (2008). Aligned Mats from Electrospun Single Fibers. Macromolecules, Vol. 41 No. 14, (June,2008), pp. 5345-5349, ISSN: 0024-9297

Chang Z, (2011). "Firecracker-shaped" ZnO/polyimide hybrid nanofibers via electrospinning and hydrothermal process. Chem. Commun., Vol. 47, (Feb, 2011), pp. 4427-4429, ISSN: 1364-548X

Chang, Z.; Xu, Y.; Zhao, X.; Zhang, Q. \& Chen, D. (2009). Grafting Poly(methyl methacrylate) onto Polyimide Nanofibers via "Click" Reaction. Vol. 1, No. 12, (Dec. 2009), pp. 2804-2811, ISSN: 1944-8252

\footnotetext{
${ }^{*}$ Corresponding Author
} 
Chen H, Song Y, Zhao Y \& Jiang L, (2008). One-Step Multicomponent Encapsulation by Compound-Fluidic Electrospray. J. Am. Chem. Soc., Vol. 130, No. 25, (May, 2008), pp. 7800-7801, ISSN: 0002-7863

Chen, D.; Liu, T.; Zhou X.; Tjiu, W. C. \& Hou, H. (2009). Electrospinning Fabrication of High Strength and Toughness Polyimide Nanofiber Membranes Containing Multiwalled Carbon Nanotubes. J. Phys. Chem. B, Vol. 113, No. 29, (July, 2009) pp. 9741-9748, ISSN: 1520-6106

Chen, F.; Peng, X.; Li, T.; Chen, S.; Wu, X.; Reneker, D. H. \& Hou, H. (2008). Mechanical characterization of single high-strength electrospun polyimide nanofibres. J. Phys. D: Appl. Phys., Vol. 41, (Jan. 2008), pp. 025308, ISSN: 0022-3727

Chen, S.; Hu, P.; Greiner, A.; Cheng C.; Cheng, H.; Chen, F. \& Hou, H. (2008). Electrospun nanofiber belts made from high performance copolyimide. Nanotechnology, Vol. 19, (Nov. 2007), pp. 015604, ISSN: 0957-4484

Cheng, C.; Chen, J.; Chen, F.; Hu, P.; Wu, X.; Reneker, D. H. \& Hou, H. (2009). High-Strength and High-Toughness Polyimide Nanofibers: Synthesis and Characterization. J. Appl. Polym. Sci.,Vol. 116, No. 3, (Jan. 2010), pp. 1581-1586, ISSN: 0021-8995

Cheng, S.; Shen, D.; Zhu, X.; Tian, X.; Zhou, D. \& Fan L. (2009). Preparation of nonwoven polyimide/silica hybrid nanofiberous fabricsby combining electrospinning and controlled in situ so-gel techniques. European Polymer Journal, Vol. 45, (July, 2009), pp. 2767-2778, ISSN: 0014-3057

Chung, G. S.; Jo, S. M. \& Kim, B. C., Properties of Carbon Nanofibers Prepared from Electrospun Polyimide, J. Appl. Polym. Sci., Vol. 97, No. 1, (Apr., 2005), pp. 165-170, ISSN: 0887-624X

Ding, M., (2011). Polyimide: monomers, polymerization and materials, Science Press, ISBN: 978-703-031080-4, Beijing, China

Dong, J. H., (2009). Frontier and Progress in Polymer Science (2nd Ed.), Science Press., ISBN: 978-7-03-023197-0, Beijing, China

Formalas A, US patent, 1975504, 1934

Gong G, Wu J. Unpublished work

Greiner A \& Wendorff J H. (2007). Electrospinning: A Fascinating Method for the Preparation of Ultrathin Fibers. Angew. Chem. Int. Ed., (June, 2007). Vol. 46, pp. 56705703, ISSN 1521-3773

Hohman M M, Shin M \& Rutledge G C, (2001). Electrospinning and electrically forced jets. II. Applications, Phys. Fluidics., (May, 2001). Vol. 13, No. 8, pp. 2201-2217, ISSN 10706631

Hou H, Reneker D H, (2004). Carbon Nanotubes on Carbon Nanofibers: A Novel Structure Based on Electrospun Polymer Nanofibers. Adv. Mater.,Vol. 16, No. 1, (Jan, 2004), pp. 69-73, ISSN 1521-4095

Huang, C.; Chen, S.; Reneker, D. H.; Lai, C. \& Hou, H. (2006). High-Strength Mats from Electrospun Poly( $p$-Phenylene Biphenyltetracarboximide) Nanofibers. Adv. Mater., Vol. 18, No. 5, (Mar. 2006), pp. 668-671, ISSN: 1521-4095 
Huang, C.; Wang, S.; Zhang, H.; Li, T.; Chen, S.; Lai, C. \& Hou, H. (2006). High strength electrospun polymer nanofibers made from BPDA-PDA polyimide. European Polymer Journal, Vol. 42, (Dec. 2005), pp. 1099-1104, ISSN: 0014-3057

Jones, R. L. \& Richards, R. W. (1999). Polymers at Surface and Interfaces, Cambridge University Press, ISBN-13: 978-0521479653, Cambridge

Kim, C.; Choi Y.; Lee, W. \& Yang, K., (2004), Supercapacitor performances of activated carbon fiber webs prepared by electrospinning of PMDA-ODA poly(amic acid) solutions. Electrochimica Acta. Vol. 50, (Aug., 2004), pp. 883-887, ISSN: 0013-4686

Lee, S. H.; Kim, S. Y.; Youn, J. R.; Seong D. G.; Jee, S. Y.; Choi, J. I. \& Lee, J. R. (2010). Processing of continuous poly(amide-imide) nanofibers by electrospinning. Polym. Int., Vol., 59, No. 2, (Feb. 2010) pp. 212-217, ISSN: 1097-0126

Li D \& Xia Y. (2004). Elctrospinning of Nanofibers: Reinventing the Wheel?. Adv. Mater., Vol. 16, No. 14, (July, 2004), pp.1151-1170, ISSN 1521-4095

Li D, Wang Y, Xia Y, (2004). Direct Fabrication of Composite and Ceramic Hollow Nanofibers by Electrospinning, Nano Lett., Vol. 4, No. 5, (Mar., 2004), pp. 933-938, ISSN: 1530-6984

Liu, J.; Min, Y.; Chen, J.; Zhou, H. \& Wang, C. (2007). Preparation of the Ultra-Low Dielectric Constant Polyimide Fiber Membranes Enabled by Electrospinning. Macromol. Rapid Commun., Vol. 28, No. 2, (Jan., 2007), pp. 215-219, ISSN: 1521-3927

Lu X, Wang C \& Wei Y, (2009). One-Dimensional Composite Nanomaterials: Synthesis by Electrospinning and Their Applications, Small, Vol. 5, No. 21, (Nov, 2009), pp. 23492370, ISSN: 1613-6810

Lv, Y.; Wu, J.; Wan, L. \& Xu, Z. (2008). Novel Porphyrinated Polyimide Nanofibers by Electrospinning. J. Phys. Chem. C. Vol. 112, No. 29, (Jun., 2008), pp. 10609-10615, ISSN: 0022-3654

Nah, C., Han, S. H., Lee, M. H., Kim, J. S. \& Lee, D. S., (2003). Characteristics of polyimide ultrafine fibers prepared through electrospinning. Polym Int., Vol. 52, No. 3, (Feb, 2003), pp. 429-432, ISSN: 0959-8103

Qin, C.; Wang, J.; Cheng, S.; Wang, X.; Dai, L. \& Chen G. (2009). Fluorescent performance of electrospun polyimide web mixed with hemicyanine dye. Materials Letters. Vol. 63, No. 15, (June, 2009), pp. 1239-1241, ISSN: 0167-577X

Reneker D H, Yarin A L, Fong H \& Koombhhongse S, (2000). Bending instability of electrically charged liquid jets of polymer solutions in electrospinning. J. Appl. Phys., (Jan, 2000). Vol. 87, No. 9, pp. 4531-4548, ISSN 0021-8979

Reneker, D H \& Chun, I., (1996). Nanometre diameter fibres of polymer, produced by electrospinning, Nanotechnology, Vol. 7, No. 3, (n.d.), ISSN: 0957-4484

Taylor, G. I. (1969). Electrically Driven Jets. Proc. Roy. Soc. A, ISBN: 1471-2946, London, Dec., 1969

Wikipedia Online. (n.d.). Electrospinning, Available from: http:/ /en.wikipedia.org/wiki/Electrospinning\#History

Xuyen, N. T.; Ra, E. J.; Geng, H.; Kim, K. K.; An, K. K. \& Lee, Y. H. (2007). Enhancement of Conductivity by Diameter Control of Polyimide-Based Electrospun Carbon Nanofibers. J. Phys. Chem. B, Vol. 111, No. 39, (Aug., 2007), pp. 11350-11353, ISSN: 1520-6106 
Yang, K. S.; Edie D. D.; Lim, D. Y.; Kim, Y.M. \& Choi Y.O., (2003), Preparation of carbon fiber web from electrostatic spinning of PMDA-ODA poly(amic acid) solution, Carbon, Vol. 41, No. 11, (Apr., 2003), pp. 2039-2046, ISSN: 0008-6223

Yarin A L, Koombhhongse S \& Reneker D H, (2001). Taylor cone and jetting from liquid droplets in electrospinning of nanofibers, J. Appl. Phys., (Aug, 2001). Vol. 90, No. 9, pp. 4836-4847, ISSN 0021-8979

Zhang, Q.; Wu D.; Qi S.; Wu Z.; Yang X. \& Jin R. (2007). Preparation of ultra-fine polyimide fibers containing silver nanoparticles via in situ technique. Materials Letters. Vol. 61 No. 19-20, (Jan. 2007), pp. 4027-4030, ISSN: 0167-577X

Zhao Y, Cao X \& Jiang L, (2007). Bio-mimic Multichannel Microtubes by a Facile Method. J. Am. Chem. Soc., Vol. 129, No. 4, (Jan, 2007), pp. 764-765, ISSN: 0002-7863

Zhu, J.; Wei, S.; Chen, X.; Karki, A. B.; Rutman, D.; Young, D. P. \& Guo, Z. (2010). Electrospun Polyimide Nanocomposite Fibers Reinforced with Core-Shell Fe-FeO Nanoparticles. J. Phys. Chem. B, Vol. 114, No. 19, (Apr., 2010), pp. 8844-8850, ISSN: 15206106 